\title{
Dural ectasia in the Marfan syndrome: MR and CT findings and criteria
}

Nicholas U. Ahn, $M D^{1}$, Paul D. Sponseller, $M D^{1}$, Uri M. Ahn, $M D^{1}$, Leelakrishna Nallamshetty, $B S^{1}$, Peter S. Rose, $B S^{1}$, Jacob M. Buchowski, $M D^{1}$, Elizabeth S. Garrett, $P h D^{2}$, Brian S. Kuszyk, $M D^{3}$, Elliot K. Fishman, $M D^{3}$, and S. James Zinreich, $M D^{3}$

\begin{abstract}
Purpose: To create criteria for detecting dural ectasia on MR or CT images in adult Marfan patients. Methods: Images were analyzed using a workstation. Parameters that predicted dural ectasia were included in our criteria. Results: Major criteria include: (1) width of dural sac below L5 > width above $L 4$; (2) anterior sacral meningocele. Minor criteria include: (1) L5 nerve root sleeve diameter $>6.5 \mathrm{~mm}$ and (2) S1 scalloping $>3.5$. Dural ectasia exists if 1 major or 2 minor criteria are present. Conclusion: MR and CT diagnose dural ectasia with high specificity and sensitivity. Our criteria accurately diagnose dural ectasia in adult Marfan patients. Genetics in Medicine,
\end{abstract} 2000:2(3):173-179.

Key Words: Marfan syndrome, connective tissue disease, dural ectasia, spinal deformity, diagnosis criteria

Dural ectasia is defined as a ballooning or widening of the dural sac, often associated with herniation of the nerve root sleeves out of the associated foraminae, ${ }^{1,2}$ and is associated with various conditions including Marfan syndrome, ${ }^{1-6}$ Ehlers-Danlos syndrome, ${ }^{7,8}$ neurofibromatosis 1 (NF1), ${ }^{9,10}$ and ankylosing spondylitis. ${ }^{11,12}$ Furthermore, dural ectasia can be associated with trauma, ${ }^{13}$ scoliosis or tumors, ${ }^{14,15}$ or may have no clear cause. ${ }^{16.17}$ However, it has not been extensively studied or reported outside of the Marfan syndrome. Dural ectasia is typically found in the most caudal portion of the spinal canal presumably because the CSF pressure is greatest in this region. ${ }^{1}$

Dural ectasia is common in Marfan syndrome $e^{1,2}$ and is clinically relevant in that it is a major criterion used in the clinical diagnosis of Marfan syndrome. ${ }^{18}$ Furthermore, it may be a source of severe back pain and headaches ${ }^{16,19-24}$ or even neurologic deficits ${ }^{2,4}$ in these patients, which may be correctable by surgery. ${ }^{4-6,19,20,23}$ In nearly all cases of dural ectasia in Marfan syndrome that have been shown in figures in the literature the diagnosis has been very simple and based on obvious bony erosions due to the enlarged dural sac. ${ }^{1,2}$ However, many authors describe "mild" dural ectasia without clearly defining what accounts for this classification. ${ }^{1,2,25}$ This study was undertaken to delineate more clearly guidelines for classifying a Marfan spine as having dural ectasia.

As previous authors have noted that dural ectasia can be diagnosed reliably only by MRI or CT, 1,2,4,25 we have created

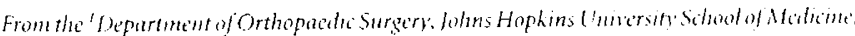
the 'Department of Oncolog), Diriston of Biostatistics, Johns Hopkins (Iniversity Sitheol of Medicine: and the 'Departinent of Rathology', Iohns Hopkins (Iniversity School of Midticine. Baltimore, Marstant

Nitholas (1. Ahn. MD, 2639 Buston St., \#2I2. Bultimorc, MI) 21224.

Received: January 31, 2000.

Accepted: Marth 30, 2000
}

criteria for dural ectasia that are easily determined from these imaging modalities. The dural volume of the normal controls was calculated and used to determine a "gold standard" for dural ectasia. However, because the volumetric criterion for dural ectasia is a novel one and requires high-powered software to calculate it (and thus, is not widely available), we developed simple criteria for evaluation of dural ectasia. These criteria were then compared against the volumetric "gold standard" to determine how accurately these simpler CT and MRI criteria could predict the presence of dural ectasia in Marfan patients.

\section{MATERIALS AND METHODS}

\section{Recruitment of volunteers}

Thirty two volunteers with Marfan syndrome as diagnosed by the Ghent criteria ${ }^{18}$ were identified either through the human genetics clinic at the Johns Hopkins Hospital, the $\mathrm{Na}$ tional Marfan Foundation, or the Marfan internet newsgroup. Exclusion criteria included failure to meet the latest criteria for Marfan syndrome and history of previous back surgery, spinal tumor, spinal stenosis, or lumbar scoliosis $>20^{\circ}$.

Two hundred and twenty two patients with a true diagnosis of Marfan syndrome were identified through patient records in the genetics clinic. Of these patients, 83 patients were between the ages of 30 and 50. Fifty one of these patients did not have a history of previous back surgery, spinal tumor, spinal stenosis, or lumbar scoliosis $>20^{\circ}$. Sixteen of these patients were available for study. The remaining sixteen patients were obtained as volunteers through the National Marfan Foundation or the Marfan internet newsgroup. Eighteen patients were female and fourteen were male. In each case the diagnosis of Marfan syndrome was reascertained through the geneticist or through the current investigators paying strict attention to the Ghent criteria as established in 1992. 
Ten additional control patients required MRI and CT scans of the lumbosacral spine for clinical indications unrelated to Marfan syndrome or other conditions in which dural ectasia has been reported. None of these patients had a diagnosis of Marfan syndrome.

This study was approved by the Johns Hopkins Hospital Joint Committee on Clinical Investigations. All study patients were appropriately consented using an approved consent form developed by our team of investigators.

\section{Radiographic evaluation}

After appropriate consent was obtained, all volunteers underwent a spiral CT scan of the lumbosacral spine at the Johns Hopkins Hospital performed without contrast using a Plus Y scanner (Siemens, Erlangen, Germany) with $3.0 \mathrm{~mm}$ collimation, $3.0 \mathrm{~mm} / \mathrm{second}$ table speed, $140 \mathrm{kVp}, 206 \mathrm{~mA}$. Images were reconstructed at $3.0 \mathrm{~mm}$ intervals. Image analysis was performed at the $\mathrm{CT}$ console using electronic calipers and multiplanar reformatted images in addition to axial sections.

Each volunteer also underwent MR scanning of the lumbosacral spine using a Signa 1.5T closed scanner (GE Medical Systems, Milwaukee, WI). The following sequences were performed: sagittal $\mathrm{T} 1$-weighted spin-echo $(\mathrm{TR}=500, \mathrm{TE}=14)$ $3.0 \mathrm{~mm}$ thick slices, $1.0 \mathrm{~mm}$ interscan gap; axial T1-weighted spin-echo $(\mathrm{TR}=500, \mathrm{TE}=14) 4.0 \mathrm{~mm}$ thick slices, $2.0 \mathrm{~mm}$ interslice gap; sagittal proton density fast spin-echo (TR = $3000, \mathrm{TE}=16) 3.0 \mathrm{~mm}$ slices, $1.0 \mathrm{~mm}$ interslice gap; sagittal $\mathrm{T} 2$-weighted $(\mathrm{TR}=2400, \mathrm{TE}=185) 1.2 \mathrm{~mm}$ slices, 0 interslice gap; and axial T2-weighted fast spin-echo $(\mathrm{TR}=4000, \mathrm{TE}=$ 96) $4.0 \mathrm{~mm}$ thick slices, $2.0 \mathrm{~mm}$ interslice gap. All sequences were $256 \times 256$ matrix, 2 NEX.

\section{Dural volume and nerve root sleeve diameter}

A Siemens workstation was used for the analyses from the MRI scans. The maximum diameter of the nerve root sleeves for each foramina at each level from L1-S1 were measured from the axial cuts. An example of this measurement is shown in Figure 1.

Dural ectasia in Marfan syndrome is associated with an enlarged dura at the most caudal aspects of the cord. ${ }^{1.2}$ Therefore, dural volume was measured caudal to the level of the inferior endplate of the L5 vertebral body. The window width and levels were adjusted to best display the anatomy and upper and lower pixel intensity thresholds set such that the dural sac was reconstructed in this "thresholded" region. Surrounding pixels within an 8 pixel region of interest (ROI) were then selected, which met the threshold limit to "grow" the dural sac. Once one region was complete, the reconstruction software automatically moved to the next slice and the growing process was continued. In this manner, the fluid volume (not including the nerve roots) in the dural sac could be accurately calculated in the sacral spine. This method of "growing" back digitized images to reconstruct an area of interest has been used widely in the literature. ${ }^{26,27}$

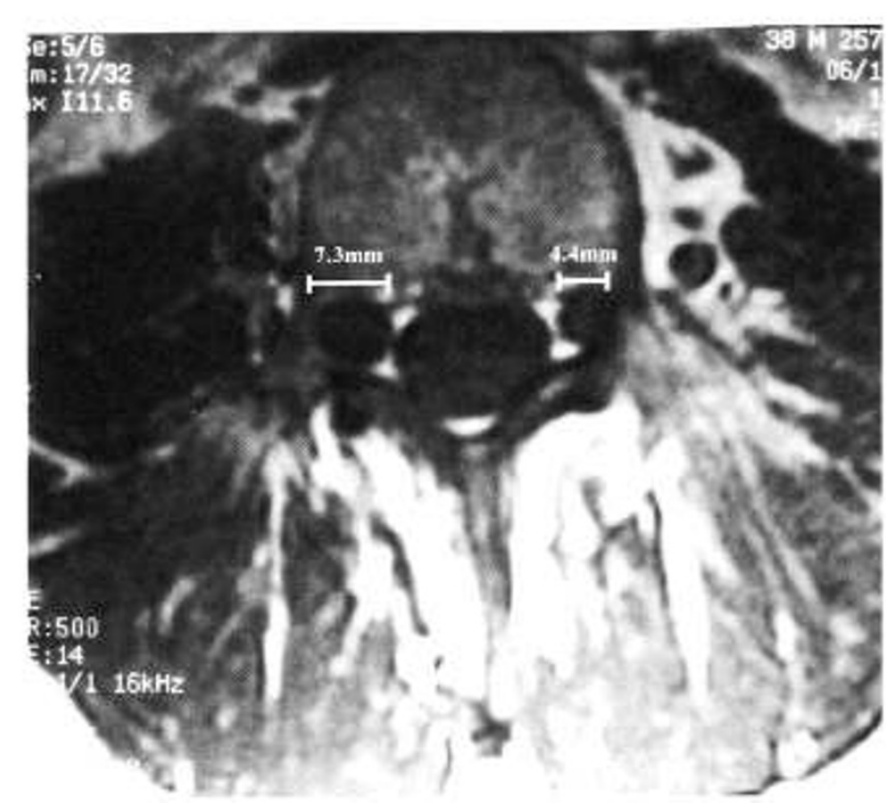

Fig. 1 Measurement of the maximum nerve root sleeve diameter at L5. Note that the nerve root sleeve diameter is $7.3 \mathrm{~mm}$ on the left and $4.5 \mathrm{~mm}$ on the right. The maximum value at $L 5$ is thus $7.3 \mathrm{~mm}$ and this meets one of the "minor" criteria. The "minor" criteria for nerve root sleeve diameter at $L 5$ is met only if the maximum value exceeds $6.5 \mathrm{~mm}$.

\section{Grouping of patients}

Dural ectasia was defined as a dural volume that was greater than two standard deviations above the mean value for the normal controls, rounded to the nearest cubic centimeter. This volume was determined to be $7.0 \mathrm{~cm},{ }^{3}$ thus any patients with a dural volume greater than this "cutoff value" were considered to have dural ectasia. In other words, because the "cutoff value" exceeded the upper limit of the $95 \%$ confidence interval for the normal controls, the specificity of using a dural volume $>7.0 \mathrm{~cm}^{3}$ as a screening tool was $95 \%$.

Patients were divided into three groups. Group I contained patients with Marfan syndrome and dural ectasia; group II consisted of patients with Marfan syndrome but without dural ectasia; and group III consisted of the control patients.

\section{Scalloping}

Scalloping has been previously defined as central erosion of the vertebral body as seen in the sagittal plane. Scalloping, presumably caused by the ballooning of the dural sac, has been noted to be associated with dural ectasia in previous reports. ${ }^{8,28.29}$ Scalloping of $\mathrm{S} 1$ was noted to be associated with dural ectasia by our initial analysis and was thus used as a minor criterion. The value was calculated by measuring the superior sagittal width, the inferior sagittal width, and the midsagittal width of S1. The superior and inferior widths were added and divided by 2 and then the midsagittal width was subtracted from this value (see Fig. 2).

\section{Initial analysis}

Three of the orthopedists (NUA, PDS, UMA) and three of the radiologists ( $\mathrm{BK}, \mathrm{EKF}, \mathrm{SIZ}$ ) convened to look at the images 

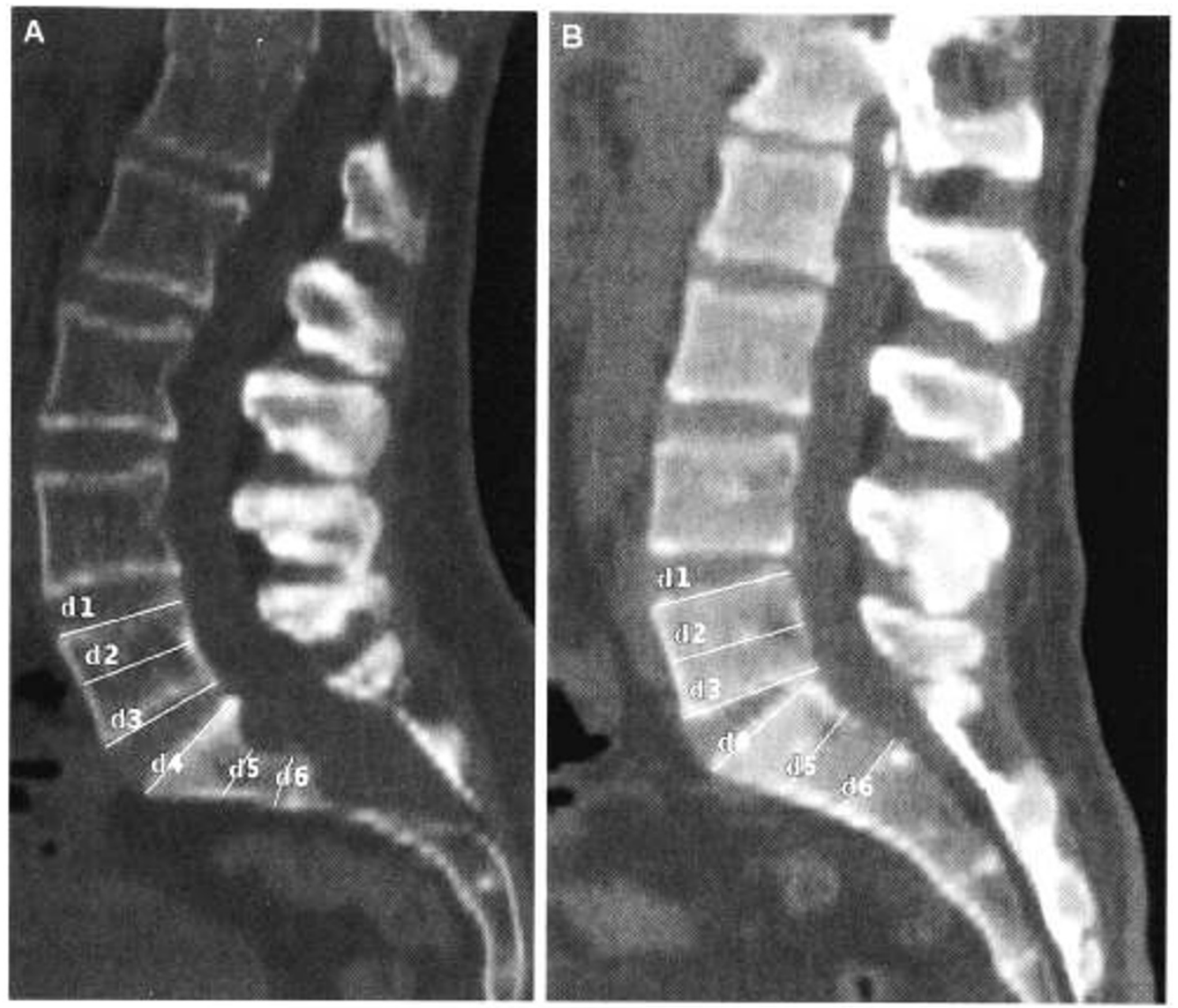

Fig. 2 Measurement of scalloping. A midline sagittal reconstruction from the spiral CT scan of a Marfan patient with clear evidence of dural ectasia is shown in a. Scalloping is measured by taking the average of the widths of the superior and inferior endplates of the vertebral bodies and subtracting the width at the midsection of the vertebral body. Thus, in this case the scalloping value at $\mathrm{L} 5$ would be [(distance $1+$ distance 3$) / 2]$ - distance 2 . The scalloping value at $\mathrm{S} 1$ would be $[($ distance $4+$ distance 6$) / 2]-$ distance 5 . The scalloping value at $\mathrm{L} 5$ is $2.3 \mathrm{~mm}$ and the scalloping value at $\mathrm{S} 1$ is $4.2 \mathrm{~mm}$ in this case. A midline sagittal reconstruction from the spiral CT scan of a Marfan patient without dural ectasia is shown in $b$. The scalloping value at L5 is $1.6 \mathrm{~mm}$ and the scalloping value at $S 1$ is $0.4 \mathrm{~mm}$ in this case.

for each patient. The reviewers were blinded as to whether or not each patient has Marfan's Syndrome. The images were assessed, and in each case a consensus as to whether dural ectasia was present or not was made. Criteria were developed that aided in differentiating those patients with and without dural ectasia. These criteria were noted. Once all of the cases had been reviewed, cases with dural ectasia were again checked to make sure that the criteria that had been developed would verify the presence of dural ectasia.

It was noted that the spinal canal was enlarged in patients with dural ectasia primarily in the most caudal portions of the canal, i.e., in the sacral spine. It was for this reason that volumetric measurements referred to above were taken below the level of L5. Similarly, the criteria that were generated referred to this caudal portion of the spine as well.

\section{Statistical analysis}

The volumetric criterion of dural volume $>7.0 \mathrm{~cm}^{3}$ was taken as the "gold standard" for presence of dural ectasia in adults. The criteria that the investigators had derived were then used to determine whether or not dural ectasia was present without knowledge of the dural volume. MRI and CT scans were assessed independently of one another. These criteria were tested against the volumetric criteria using logistic regression. Sensitivity, specificity, positive predictive value and negative predictive values were derived, and an ROC (receiver operating characteristic) curve was created to determine how well our criteria predicted excessive dural volume and thus dural ectasia (in other words, the ROC curve was used to assess the ability of our criteria to discriminate between those with and without dural ectasia).

\section{RESULTS}

\section{Demographics}

Thirty two patients with Marfan syndrome and 10 control patients were analyzed. The controls were matched to the Marfan subjects with respect to age and sex. The average age of the controls was 40.1 years with a standard deviation of 4.2 years; the average age of the Marfan patients was 38.9 years with a standard deviation of 5.7 years. A t-test comparing the mean ages between the two groups demonstrates that there is no significant difference ( $P=0.54$ ). Fifty percent of the control patients ( 5 of 10 ) were male and $54.5 \%$ of the Marfan patients (18 of 33) were male. A Fisher's exact test demonstrates that 
there is no significant difference in the proportion of male patients in each group $(P=0.80)$.

Twenty patients with Marfan syndrome were noted to have dural ectasia and 12 did not have dural ectasia. These patients were put into group I (dural ectasia) and group II (Marfan and no dural ectasia), respectively. The 10 control patients without Marfan syndrome were placed into a separate group III.

The mean dural volume was $38.12 \mathrm{~cm}^{3}$ in group $1,6.55 \mathrm{~cm}^{3}$ in group II, and $3.67 \mathrm{~cm}^{3}$ in group III. This data, with the $95 \%$ confidence intervals for the mean, is summarized in Table 1. All 10 of the control patients did not have dural ectasia by the volumetric criteria mentioned previously (i.e., dural volume $>$ $\left.7.0 \mathrm{~cm}^{3}\right)$.

\section{Criteria for dural ectasia}

The criteria that were developed included two "major" criteria and two "minor" criteria. If a patient had any one of the two "major" criteria he or she was considered to have dural ectasia; a patient needed both "minor" criteria to be considered to have dural ectasia.

The "major" criteria were: sagittal diameter of spinal canal at S1 or below greater than sagittal diameter at midaxis of L4 or above; and presence of anterior meningocele. "Minor" criteria were obtained by noting that the scalloping at S1 and the maximum L5 nerve root sleeve diameter were consistently larger for patients with generous dural sacs. S1 nerve root sleeve diameter was not used because measurements were inconsistent. Means and standard deviations were obtained for the normal controls and an individual was considered to have a positive "minor" criterion if the value for scalloping at S1 or maximum nerve root sleeve diameter at L5 exceeded a "cutoff value" defined as two standard deviations greater than the mean for the controls. MRI was used to determine the "cutoff value" for nerve root sleeve diameter at L5 because MR is more capable of measuring soft tissue structures; CT was used to determine the "cutoff value" for scalloping because CT scanning is superior for making bony measurements. The mean value for maximum nerve root sleeve diameter at L5 for the controls was 5.14 $\mathrm{mm}$ with a standard deviation of $0.63 \mathrm{~mm}$; the mean value for scalloping at $\mathrm{Sl}$ for controls was $2.55 \mathrm{~mm}$ with a standard deviation of $0.48 \mathrm{~mm}$. Thus, the "cutoff values" were taken as $6.5 \mathrm{~mm}$ for maximum nerve root sleeve diameter at L5 and 3.5

Table 1

Mean dural volumes and $95 \%$ confidence intervals for patients in groups I, II, and III

\begin{tabular}{lccc}
\hline & $\begin{array}{c}\text { Population } \\
\text { Size (N) }\end{array}$ & $\begin{array}{c}\text { Mean Dural } \\
\text { Volume } \\
\left(\mathrm{cm}^{3}\right)\end{array}$ & $\begin{array}{c}95 \% \mathrm{CI} \\
\left(\mathrm{cm}^{3}\right)\end{array}$ \\
\hline $\begin{array}{c}\text { Patients with MFS } \\
\text { and DE (Group I) }\end{array}$ & 20 & 38.12 & $25.07-51.18$ \\
$\begin{array}{c}\text { Patients with MFS but } \\
\text { not DE (Group II) }\end{array}$ & 12 & 6.55 & $0.00-13.97$ \\
$\begin{array}{c}\text { Control Patients } \\
\text { (Group III) }\end{array}$ & 10 & 3.67 & $1.79-4.35$ \\
\hline
\end{tabular}

$\mathrm{mm}$ for scalloping at $\mathrm{S} 1$. Table 2 illustrates the mean scalloping values and $95 \%$ confidence intervals for scalloping in each of the three different groups.

\section{Validation of criteria: statistical analysis}

Evaluation of CT scans (Fig. 3) using our criteria compared with the volumetric criteria yielded a sensitivity of $82.6 \%$, a specificity of $90.0 \%$, a positive predictive value of $90.5 \%$, and a negative predictive value of $81.8 \%$. The false positive rate was $10.0 \%$ and the false negative rate was $17.39 \%$. The ROC curve was generated to assess the ability of our criteria to discriminate between patients with and without dural ectasia, and it was determined that the area under the curve was 0.8630 .

Evaluation of MRI scans (Fig. 4) using our criteria compared with the volumetric criteria yielded a sensitivity of $87.0 \%$, a specificity of $95.0 \%$, a positive predictive value of $95.2 \%$, and a negative predictive value of $86.4 \%$. The false positive rate was $5.0 \%$ and the false negative rate was $13.04 \%$. The ROC curve was generated and the area under the curve was 0.9098 .

When the major criteria alone were used (thus obviating the need to make measurements for scalloping and/or nerve root sleeve diameters at L5) the classification of dural ectasia did not differ in any patient between the CT and MRI scans. Therefore, the statistical analysis comparing the major criteria alone versus the volumetric "gold standard" was the same using MRI and CT. We noted a sensitivity of $79.17 \%$, a specificity of $100 \%$, a positive predictive value of $100 \%$, and a negative predictive value of $79.17 \%$. The false positive rate was $0 \%$ and the false negative rate was $20.8 \%$. An ROC curve could not be calculated because positive major criteria perfectly predicted "true" dural ectasia. These data are summarized in Table 3.

\section{DISCUSSION}

Dural ectasia has been widely reported in the literature in numerous conditions and syndromes but the radiologic criteria have never been described. This is particularly concerning when authors discuss "mild" amounts of dural ectasia as opposed to severe dural ectasia. ${ }^{1,2,25}$ In fact, all of the images of dural ectasia that are present in the literature are "severe" and obvious cases in which there is no diagnostic dilemma. 1,2,16,21,23 Clinical relevance lies in the fact that dural ectasia is considered

Table 2

Mean scalloping values and $95 \%$ confidence intervals for patients in groups I, II, and III

\begin{tabular}{lccc}
\hline & $\begin{array}{c}\text { Population } \\
\text { Size (N) }\end{array}$ & $\begin{array}{c}\text { Mean Scalloping } \\
\text { Value (mm) }\end{array}$ & $\begin{array}{c}95 \% \mathrm{CI} \\
(\mathrm{mm})\end{array}$ \\
\hline $\begin{array}{c}\text { Patients with MFS } \\
\text { and DE (Group I) }\end{array}$ & 20 & 7.85 & $4.41-11.29$ \\
$\begin{array}{c}\text { Patients with MFS but } \\
\text { not DE (Group II) }\end{array}$ & 12 & 3.12 & $1.49-4.76$ \\
$\begin{array}{c}\text { Control Patients } \\
\text { (Group III) }\end{array}$ & 10 & 2.55 & $1.45-3.65$ \\
\hline
\end{tabular}



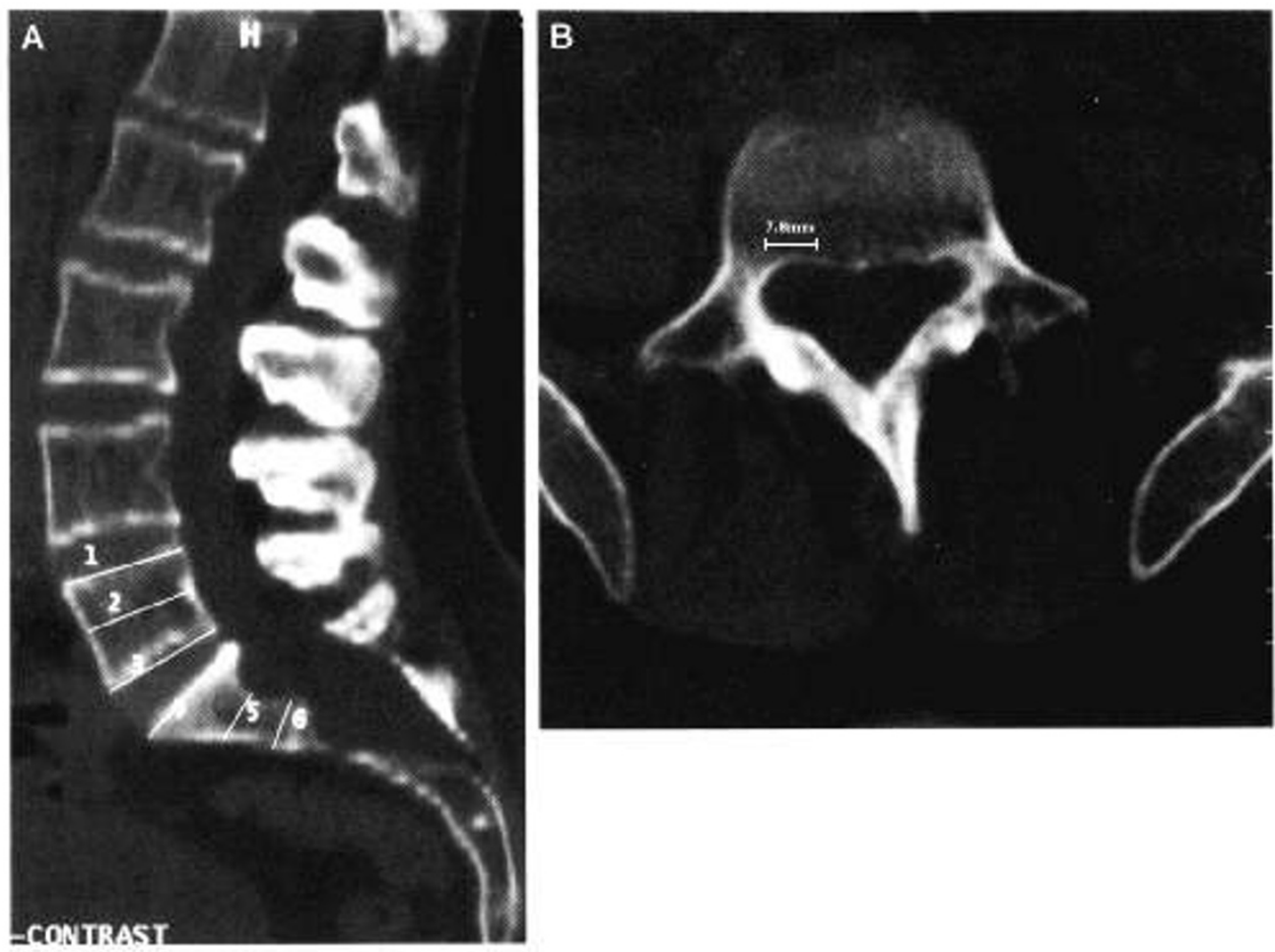

Fig. $3 \mathrm{CT}$ scan demonstrating dural ectasia in a patient with Marfan syndrome. A sagittal midline reconstruction is shown in $a$. Note that the spinal canal is wider at S2 than at $\mathrm{L} 4$. Scalloping value at $\mathrm{S} 1$ was calculated to be $4.2 \mathrm{~mm}$. Evaluation of axial slice through L5 $(b)$ demonstrates a nerve root sleeve diameter equal to $6.6 \mathrm{~mm}$. This patient meets one major and two minor criteria.

a major diagnostic criterion for Marfan syndrome ${ }^{18}$ and yet from our experience the presence of dural ectasia is not always clear. Rose et al. ${ }^{30}$ found that dural ectasia constituted the second major diagnostic manifestation of Marfan syndrome (without which the diagnosis would not be supported) in 9 of $39(23 \%)$ patients meeting the Ghent diagnostic criteria. In that study, dural ectasia was the second most common major diagnostic manifestation following aortic dilatation/dissection in a population of Marfan patients. Thus, despite the added cost of imaging, the presence of dural ectasia is often required to diagnose Marfan syndrome. Furthermore, dural ectasia may be a cause of back pain and headaches ${ }^{19-24}$ or neurologic deficits $^{2,4}$ in these patients; in severe cases, surgical decompression may be warranted. ${ }^{4-6,19,20,23}$ However, indications and techniques for decompression have not been established and no long-term follow-up of patients with history of decompression has been reported. Furthermore, the natural history of dural ectasia has not been well described. Thus, the ability to detect dural ectasia has important implications for diagnosis, treatment, and research in Marfan syndrome.

The criteria we developed for determining whether or not dural ectasia was present are listed in Table 4 . These criteria were developed to give a simple means of determining whether dural ectasia is present in the adult Marfan patient because the authors realize that most readers will not have access to volume calculating software. The "gold standard" for dural ectasia was taken as a dural volume that was greater than two standard deviations above the mean for the normal controls rounded up the nearest cubic centimeter. This "cutoff value" was thus 7.0 $\mathrm{cm}^{3}$, and none of the normal controls had a dural volume greater than this value. When evaluation of CT scans using our criteria was compared against this volumetric "gold standard," the sensitivity exceeded $80 \%$ and specificity was $90 \%$. The ROC curve demonstrated an area of 0.8630 showing that CT could accurately detect dural ectasia. However, MRI was superior with a sensitivity exceeding $85 \%$ and a specificity of $95 \%$. The ROC curve demonstrated a larger area of 0.9098 . Therefore, while CT is an accurate means of determining the presence of dural ectasia, MRI provides superior imaging and no radiation exposure. This improved accuracy and safety needs to be weighed against the increased cost of MRI.

We felt it important to assess the validity of using the major criteria alone, since these criteria are easiest to employ because they do not require measuring nerve root sleeve diameters or vertebral scalloping. If the major criteria alone were used, the classification of dural ectasia did not differ if MRI or CT was used. While the specificity improved to $100 \%$, the sensitivity decreased to below $80 \%$. Furthermore, while the false positive rate decreased to $0 \%$, the false negative rate increased to $20.8 \%$. Thus, while use of the major criteria alone obviates the need to make direct measurements, an increased false negative rate is noted. We feel that making the measurements for the minor criteria is a useful addition.

It should be mentioned that in all cases of our Marfan patients with dural ectasia, the enlarged sac was noted primarily below the level of L5. Previous authors have noted the same 

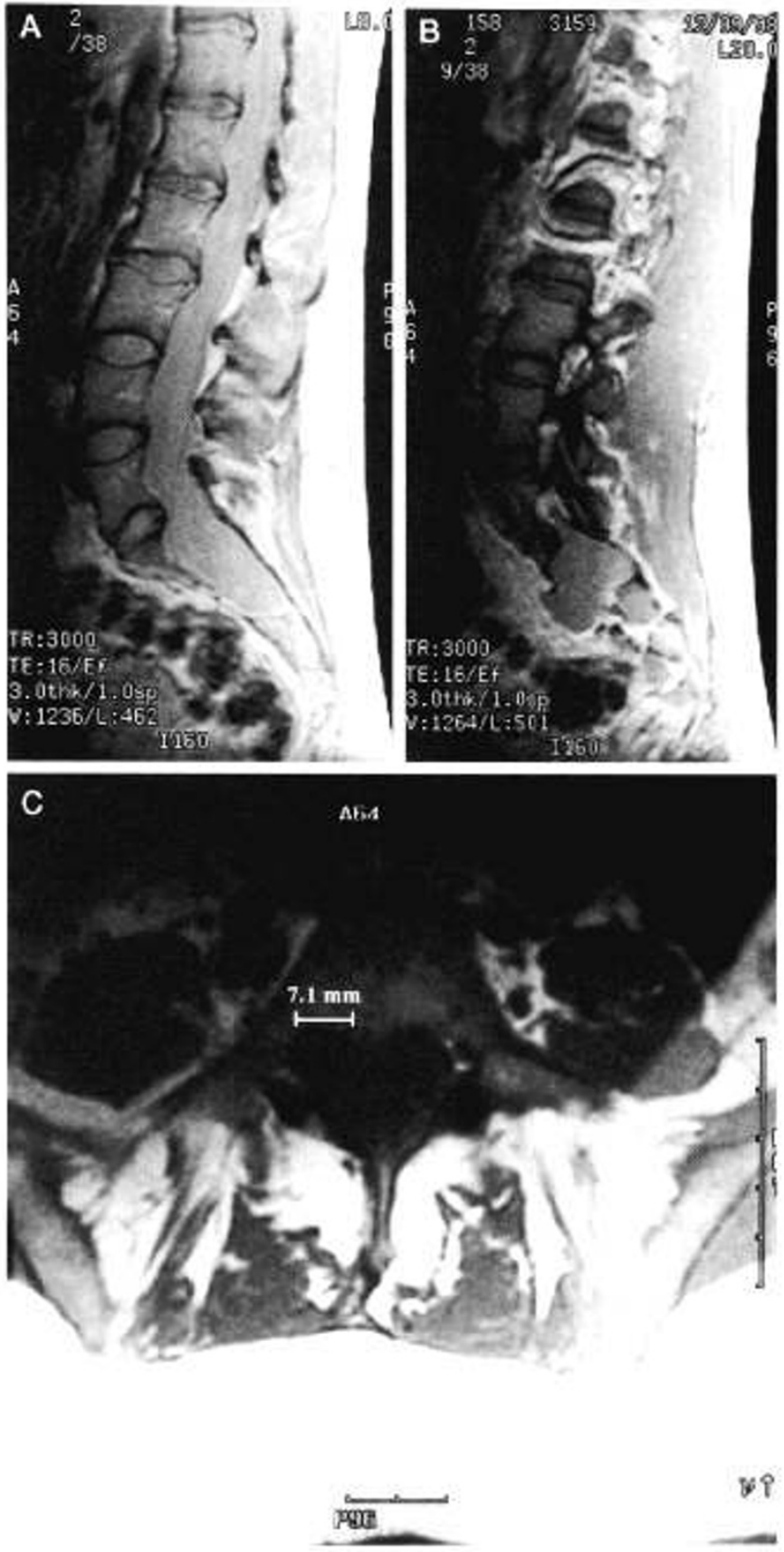

Fig. 4 MRI scan demonstrating dural ectasia in a patient with Marfan syndrome. A sagittal midline T2 weighted image ( $a$ ) clearly demonstrates that the diameter of the dural $\mathrm{sac}$ is wider at $\mathrm{S} 2$ than at L4. A large anterior sacral meningocele is present. Scalloping value at $S 1$ was calculated to be 5.1 . Axial slice through $L 5(b)$ demonstrates a nerve root sleeve diameter $>7.0 \mathrm{~mm}$. This patient meets both major and both minor criteria.

finding. ${ }^{1}, 2,4,5,19,23,31,32$ This is consistent with the hypothesis that the weakened connective tissues associated with Marfan syndrome is the cause for the formation of ectasias because the fluid pressure in the most caudal portion of the spine should be the greatest. Therefore, all measurements for dural volume were made below the level of L5, and the criteria we generated all referred to the most caudal portions of the spine.

We do realize that the number of controls in our study is relatively small. However, the standard deviation for the dural
Table 3

Validation of criteria for dural ectasia on MR and CT

\begin{tabular}{lccccc}
\hline & \multicolumn{2}{c}{ CT } & & \multicolumn{2}{c}{ MR } \\
\cline { 2 - 3 } \cline { 5 - 6 } & Major & $\begin{array}{c}\text { Major }+ \\
\text { Minor }\end{array}$ & & Major & $\begin{array}{c}\text { Major }+ \\
\text { Minor }\end{array}$ \\
\hline Sensitivity & 79.2 & 82.6 & 79.2 & 87.0 \\
Specificity & 100.0 & 90.0 & 100.0 & 95.0 \\
Positive predictive value & 100.0 & 90.5 & 100.0 & 95.2 \\
Negative predictive & 79.2 & 81.8 & 79.2 & 86.4 \\
$\quad$ value & & & & \\
False positive rate & 0.00 & 10.0 & & 0.00 & 5.00 \\
False negative rate & 20.8 & 17.4 & & 20.8 & 13.0 \\
ROC & $*$ & 0.863 & $*$ & 0.909
\end{tabular}

* ROC curve could not be generated as major criteria alone "perfectly" predicted dural ectasia.

Table 4

The MR and CT criteria for dural ectasia in Marfan syndrome

Presence of dural ectasia Presence of ONE major criterion OR presence of BOTH minor criteria

Major criteria

Saggital width of dural sac at $S 1$ or below greater than width of dural sac above L4

Presence of anterior menigocele

Minor criteria

Nerve root sleeve at $\mathrm{L} 5>6.5 \mathrm{~mm}$ in diameter

Scalloping at $\mathrm{S} 1>3.5 \mathrm{~mm}$

volumes in the control group is very small $\left(\mathrm{SD}=1.7 \mathrm{~cm}^{3}\right.$ ) demonstrating that the control group displayed a tight distribution for dural volumes. Thus, only a small number of individuals was needed to attain a high precision for dural volumes in this control group.

In summary, criteria were developed to easily determine whether or not dural ectasia is present on CT and MRI in adult patients without the need for advanced imaging software. These criteria were validated by comparing results with a volumetrically defined "gold standard." We believe that this information is clinically relevant because dural ectasia is a major diagnostic criterion in Marfan syndrome and because dural ectasia may be responsible for serious symptoms in these patients. Although we have developed criteria to easily assess the presence of dural ectasia in adults, criteria still need to be developed for children, in whom the diagnosis is often more difficult on account of age dependency.

\section{References}

1. Fishman EK, Zinreich SJ, Kumar AJ, Rosenbaum AE, Siegelman SS. Sacral abnormalities in Marfan syndrome. / Comp Assist Tomogr 1983;7:851-856.

2. Pyeritz RE, Fishman EK, Bernhardt BA, Siegelman SS. Dural ectasia is a common feature of the Marfan syndrome. Am J Hum Genet 1988;43:726-732.

3. Bean WB, Fleming JG. Arachnodactyly: report of a case complicating pregnancy at term. Ohio State Med J 1940;36:155-163.

4. Stern WE. Dural ectasia and the Marfan syndrome. J Neurosurg 1988;69:221-227. 
5. Schneider MB, Dittmar S, Boxer RA. Anterior sacral meningocele presenting as a pelvic/abdominal mass in a patient with Marfan syndrome. J Adolesc Health 1993; 14:325-328.

6. Smith MD. Large dural defect in Marfan syndrome. J Bone Joint Surg 1993;75A: 1067-1070.

7. MacFarlene IL. Ehlers-Danlos syndrome presenting certain unusual features. / Bone Joint Surg 1959;41B:541-545.

8. Mitcell GE, Lourie H, Berne AS. The various causes of scalloped vertebrae with notes on their pathogenesis. Radiology 1967;89:67-74.

9. Casselman ES, Mandell GA. Vertebral scalloping in neurofibromatosis. Radiology 1979;131:89-94.

10. Eichhorn C, Wendt G, Staudte HW, Gilsbach JM. Dural ectasia in von Recklinghausen's disease of the lumbar spine: a case report. J Bone Joint Surg 1995;77B:834835

11. Abello R, Rovira M, Sanz MP, Capdevila A, Escalada J, Peri J. MRI and CT of ankylosing spondylitis with vertebral scalloping. Neuroradiol 1988;30:272-275.

12. Sparkling MJ, Bartleson JD, McLeod RA, Cohen MD, Ginsburg WW. Magnetic resonance imaging of arachnoid diverticula associated with cauda equina syndrome in ankylosing spondylitis. / Rheum 1989;16:1335-1337.

13. Maiuri F, Corriero G, Gianpaglia F, Simonetti L. Lateral thoracic meningocele. Surg Neurol 1986:26:409-412.

14. Fiandaca MS, Ross WK, Pearl GS, Bakay AE. Carcinoid tumor in a presacral teratoma associated with an anterior sacral meningocele: case report and review of the literature. Neurosurgery 1998;22:581-589.

15. Schwend RM, Hennrikus W, Hall JE, Emans JB. Childhood scoliosis: clinical indications for magnetic resonance imaging. / Bone Joint Surg 1995;77A:46-53.

16. Cilluffo JM, Gomex MR, Reese DF. Idiopathic ("congenital") spinal arachnoid diverticula. Mayo Clin Proc 1981;56:93-101.

17. Barnes PD, Brody JD, Jaramillo D, Akbar JU, Emans JB. Atypical idiopathic scoliosis: MR imaging evaluation. Radiology 1993;186:247-253.

18. DaPaepe A, Devereux RB, Dietz H, Hannekam RCM, Pyeritz R. Revised diagnostic criteria for the Marfan syndrome. Am J Med Genet 1996;62:417-426.
19. Strand RD, Eisenberg HM. Anterior sacral meningocele in association with Marfan's syndrome. Radiology' 1970;99:653-654.

20. Weir B. Leptomeningeal cysts in congenital ectopia lentis: case report. $J$ Neurosurg 1973;38:650-654.

21. Arroyo JF, Garcia JF. Case report 582. Skel Radiol 1989;18:614-618.

22. Robinson L, Dominguez R, Cabrera J, Yeakley JW, Fenstermacher MJ, Milner ME. Multiple meningeal cysts in Marfan syndrome. Am J Neuroradiol 1989;10:12751276.

23. Raftopoulos C, Pierard GE, Retif C. Braude P, Brotchi J. Endoscopic cure of a giant sacral meningocele associated with Marfan's syndrome: case report. Neurosurg 1992; 30:765-768.

24. Duncan RW, Esses S. Marfan's syndrome with back pain secondary to pedicular attenuation: a case report. Spine 1995;20:1197-1198.

25. Soulen RL, Fishman EK, Pyeritz R. Marfan syndrome: evaluation with MR imaging versus CT. Radiology 1987;165:697-701.

26. Yoshimura H, Giger ML, Doi K, Ahn N, McMahon H. Use of morphological filters in the computerized detection of lung nodules in digital chest images. Radiology 1989;173P:347.

27. Giga ML, Ahn N, Doi K, McMahon H, Metz CE. Computerized detection of pulmonary nodules in digital test images: use of morphological filters in reducing falsepositive detections. Medical Physics 1990;17:861-865

28. Nelson JD. The Marfan syndrome with special reference to congenital enlargement of the spinal cord. Br J Radiol 1958;31:561-564.

29. Kohlmeier L, Gasner C, Bachrach LK, Marcus R. The bone mineral status of patients with Marfan syndrome. J Bone Min Res 1995;10:1550-1555.

30. Rose P, Levy H, Ahn N, Sponseller P, Magyari T, Davis J, Francomano C. A comparison of the Berlin and Ghent Nosologies in the diagnosis of Marfan syndrome: the NIH experience. Genet Med 2000;2:86-86.

31. Schlesiger EB. The significance of genetic contributions and markers in disorders of spinal structure. Neurosurg 1990;26:944-951.

32. Sonier CB, Buhe T, Despins PH, Delumeau J, Kersaint-Gilly AD. Sacral pseudomeningocele and Marfan's disease: one case. / Neuroradiol 1993;20:292-296. 